\section{Origin of the Third Heart Sound}

For many years there has been speculation about the origin of the third heart sound. Some authors have held the theory that it is the sound of ventricular filling. ${ }^{1-3}$ Others have considered the sound to originate from the mitral valve, whether representing early closure of the valve ${ }^{4}$ or due to sudden tautening of the valve and its chordal attachments in early diastole. ${ }^{6}{ }^{7}$

Recently J. S. Fleming, ${ }^{8}$ in a study of mitral regurgitation before and after mitral valve replacement, produced evidence suggesting that the third heart sound in this condition may be due to sudden tautening of the valve in early diastole. Ultrasound measurements indicated that at the time of the sound the valve was in the half-open position, while the apex cardiogram showed abrupt checking of outward movement of the apex of the heart. He suggested that the latter is due to a suddent limitation of downward movement imposed by the tautened mitral valve with its chordal attachments. After excision of the mitral valve and replacement by a StarrEdwards prosthesis the third sound disappeared, and apex cardiography no longer showed a checking movement in early diastole. This might support the theory that intact chordae tendineae are necessary for the production of the third heart sound. Disappearance of the sound might alternatively have resulted from the relief of mitral incompetence, with abolition both of the abnormally large left atrioventricular gradient in early diastole and of the increased stroke output of the left ventricle.

Different combinations of factors in different conditions may contribute to the variable nature of the third heart sound. When the mitral valve is diseased from rheumatism and the chordae tendineae thickened and shortened, it seems possible that sudden tensing of the valve apparatus may be an important factor in the production of the third sound, which in this condition may be exceptionally loud and sharp in character. In severe aortic incompetence closure of the mitral valve in early-to-mid-diastole has been shown to occur, ${ }^{9-11}$ and this may in part be responsible for a loud third sound that may persist for many years. In constrictive pericarditis, on the other hand, where a special knocking form of third heart sound occurs, full dilatation of the ventricle is prevented. It is unlikely, therefore, that the third heart sound in this condition is produced by tautening of the chordae. It is more probable that it is a water-hammer effect resulting from sudden arrest, by the rigid pericardial casing, of rapid early ventricular filling consequent on the steep atrioventricular gradient. ${ }^{12}$ The dull, low-pitched third heart sound heard in hypertensive heart failure may well be no more than the reflection of a raised left atrioventricular pressure gradient in

1 Potain, P. C. E., Semaine Médicale, 1900, 20, 175.

- Crevasse, L., Wheat, M. W., Wilson, J. R., Leeds, R. F., and Taylor, W. J., Circulation, 1962, 25, 635 .

- Kuo, P. T., Hildreth, E. A., and Kay, C. F., Annals of Internal Medicine, $1951,35,1306$.

- Gibson, A. G., Lancet, 1907, 2, 1380.

- Thayer, W. S., Archives of Internal Medicine, 1909, 4, 297

- Dock, W., Circulation, 1959, 19, 376.

' Nixon, P. G. F., British Heart fournal, 1961, 23, 677.

- Fleming, J. S., British Heart fournal, 1969, 31, 192.

- Wright, J. L., Toscano-Barboza, E., and Brandenburg, R. O., Proceedings of the Staff Meetings of the Mayo Clinic, 1956, 31, 120.

Welch, G. H., jun., Braunwald, E., and Sarnoff, S. J., Circulation Research, 1957, 5, 546.

1 Dodge, H. T., Sandler, H., and Evans, T., Circulation, 1960, 22, 741.

12 Mounsey, P., British Heart fournal, 1955, 17, 143. 12 Mourdiinis, Ath., Olsen, E., Raphael, M. J., and Mounsey, J. P. D., early diastole-in fact, a mild water-hammer phenomenon similar to that in constrictive pericarditis.

Still another type of third heart sound is that heard in ventricular aneurysm, which may persist for many years and does not necessarily carry a poor prognosis. Possibly an important factor here may be the thinned portion of ventricular wall transmitting vibrations within the chamber more freely to the exterior. ${ }^{13}$ Finally, the third heart sound in healthy persons under 40 and its disappearance after this age are phenomena whose mechanism is not fully understood. There is evidence that myocardial relaxation is an active process, the ventricle sucking in blood in early diastole. It is possible that the sequential interaction of the forces of active and passive ventricular filing is another factor that may contribute to the production of the third heart sound in health and also in disease.

\section{Cancer and the Nervous System}

A glaring gap in our knowledge is the mechanism of generalized wasting associated with malignant neoplasia. A lead may come from studying the distant effects of carcinoma on the neuromuscular system, pioneered by the late Lord Brain and colleagues, who named this group of conditions the carcinomatous neuromyopathies. ${ }^{1}$ It is fitting that this research continues at the London Hospital, ${ }^{2}$ where many of them were delineated.

A carcinoma or reticulosis may, without secondary deposits, profoundly affect neurones, myelin, muscle, or the myoneural junction. The diversity of clinical syndromes include a myopathy, a myasthenic syndrome, a sensory or mixed motor and sensory neuropathy, anterior-horn cell disease mimicking motor neurone disease, subacute cerebellar atrophy, and progressive leucoencephalopathy. The central or peripheral nervous system may be affected at various levels, singly or in combination. These neurological syndromes are not rare. Brain and R. A. Henson ${ }^{1}$ estimated an incidence of $6 \%$ in all patients with a carcinoma. About half the affected patients have neurological symptoms or signs before the diagnosis of neoplasm has been made. Unfortunately removal of the growth, even completely, often fails to reverse the neurological damage. ${ }^{2}$

Carcinomatous myopathy affects the muscles of the limb girdles and trunk. Patients have difficulty in turning in bed, rising from the lying or sitting position, and negotiating stairs, because trunk and pelvic-girdle muscles are weak. Combing hair or hanging washing on a line may become impossible for a woman or shaving for a man because of weakness in the shoulder muscles. Muscular fatigue at times responds, partially or completely, to treatment with an anticholinesterase and hence resembles myasthenia gravis. Clinically there is a close resemblance to thyrotoxic myopathy, myasthenia gravis, dermatomyositis, or polymyositis. The diagnosis of carcinomatous myopathy should be considered when the patient also has dysaesthesiae or the tendon reflexes are depressed beyond the field of weakness. ${ }^{1}$

A pure sensory neuropathy was first described by $D$. Denny-Brown. ${ }^{3}$ Neurones of the posterior root ganglia are primarily affected, with consequent degeneration of the peri- 